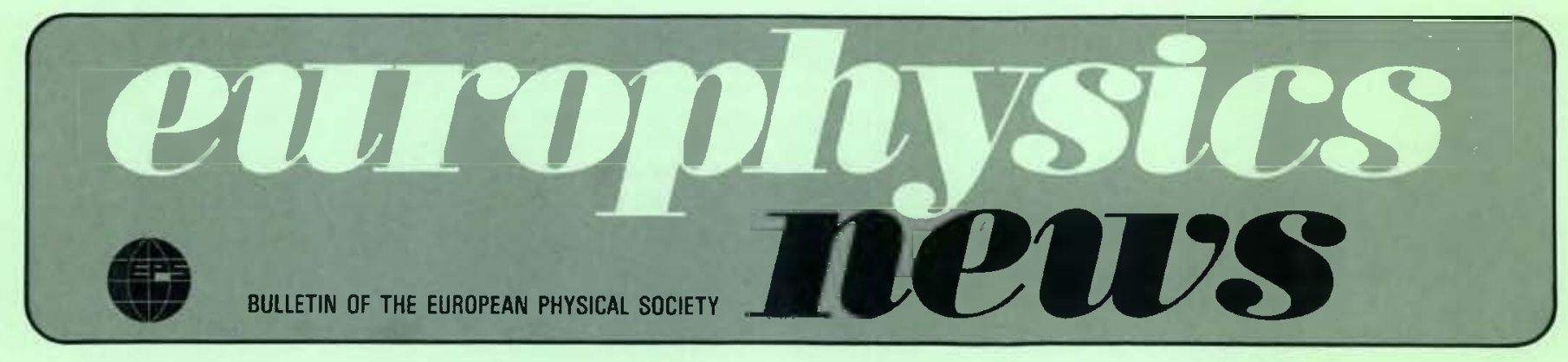

\title{
CUMulative INDEX 1981-1985
}

ISSUES AND SPECIAL FEATURES

\begin{tabular}{|c|c|c|c|c|c|c|}
\hline No. & Vol. 121981 & Vol. 131982 & Vol. 141983 & Vol. 151984 & Vol. 161985 & Month \\
\hline 1 & * & * Solar Physics & * Metal Physics & * Nuclear Physics & * Atomic \& Mol. Physics & January \\
\hline 2 & * & * & * & * & * & February \\
\hline 3 & Meetings & Meetings & Meetings & Meetings & Meetings & March \\
\hline 4 & * & * & * & * & * & April \\
\hline 5 & * & * & * & * & * & May \\
\hline 6 & Meetings \& Directory & Meetings \& Directory & Meetings \& Directory & With Directory & ${ }^{*}$ With Directory & June \\
\hline 7 & * Lasers & * & * & * & & July \\
\hline 8 & & ( & f & & \} & August \\
\hline 9 & \}$*\{$ & \}$*\{$ & * & * \{ & * & September \\
\hline 10 & * & * & * & Meetings & Meetings & October \\
\hline 11 & Meetings & Meetings & Meetings & 21 & ) & November \\
\hline 12 & $*$ & * & * & & & December \\
\hline
\end{tabular}

* Contributed articies

References include vol. no. (bold) issue (ital.) and first page.

\section{PRINCIPAL SUBJECT}

Accelerator mass spectroscopy for single atom counting, 15,2,1

Applied Physics, Physics in Industry

- Chemical vapour deposition, laser-induced, 14, 10.9

- at Condensed Matter Conf. (Berlin-West 1985), 16,5.9

- GEC, long-range research at, 14,5,5

- Hydrogen production by water electrolysis, 12,8/9,9

- Laser applications, 12, 7,10; 14, 10,9

- Microemulsions, amphiphiles, micelles, 16,6,9

Optical fibre technology at STL, 16, 11/12,4

- University - industry interaction, 14,8/9,8

APS (American Phys. Soc.), collaboration with, 16, 11/12,20

Associate Members, EPS : see EPS

Astronomy, Astrophysics (see also Solar Physics)

Atomic processes in, 16,1,12

Atoms in highly ionized states, 12, 1,1

- Cosmic rays, what are they made of ?, 15,2,12

ESO new technology telescope, 13, 12,12

Extragalactic distance scale, Hubble constant, 15, 11/12,10

- Gamma-ray astronomy beyond COS-B mission, 13, 7,1

- Inflationary Universe, 16,9,6

- Neutrinos in cosmology, 14,7,1

- Neutron stars, 12,1,5

- Nobel Prize in Physics 1983, 14, 12,5

- Primordial helium, 12,8/9,7

- Solar and stellar magnetic fields, 13,12,6

- SS 433: new extraordinary object, $12,4,8$

- Supernovae and their progenitors, 16,7/8,5

- Volcanism of lo, 12, 10,9

7th European Regional Astronomy Meeting (Florence 1983), 15,4,8

Atomic and Molecular Physics

- in Collisions, 12, 1,3:16, 1,6

Dimer spectroscopy and vibrational predissociation, 16,1,9

an Exciting field of many facets, 16, 1,1

- Heavy atoms, parity non-conservation in, 16,2,1

- Ion-atom collision theory, 16,1,6

- Molecular beams, European Who's Who, 15,11/12,16

- - Multiphoton dissociation in, 16, 1,2

- Molecular systems, theory of resonances in, 16,2,4

- Special Issue, 16,1

- Structure of matter, research in Italy on, 13,4,8

- 1st European Conf. of Atomic Phys. Division 1981, 12, 7,15
Atomic Spectroscopy

- EGAS meeting, Liège 1982, 14,4,11

- Madrid 1983, 15,4,16

- Gamma-ray internal conversion for, 15,4,11

Atoms in highly ionized states, 12,1,1

Barkhausen: physicist and pioneer, 12, 12,10

BESSY, surface physics at, 15,6,1

Biophysics, Medical Sciences

- Biological tissue characterisation by ultrasound, 15,8/9,12

- Biomagnetism, 15, 7,11

- Lasers in biology and medicine, 12,7,14

- NMR tomography, 15,5,10

- from Photon to neuronal signal, 16, 5,1

Boundary and Interior Layers, BAIL Conf. Dublin 1982, 13, 12,6

Career outlook for physicists in Europe, 12, 12,8

CERN

- ISOLDE experiments, 12,4,1

- LEAR experiments, 15,7.5

- LEP ground-breaking ceremony, 14, 10,12

- Proton-antiproton collider experiments, 14, 10,1

Computational Physics

- Boundary and interior layers (BAIL), 13,12,6

- Computations in plasma physics, 12,12,4

- EPS Group activities, 12, 12,11

- New developments, 12, 1,11

Condensed Matter Physics (see also Solid State Physics)

- Amphiphiles, micelles, microemulsions, 16.6.9

- EPS Division applauds 1985 Nobel Prize, 16, 11/12,3

- EPS Division, General Conference

- 5th Berlin-West 1985, 16,1,16; 16,5,8;16,5,9

- 3rd Lausanne 1983, 14,7,6

- 2nd Manchester 1982, 13,2,12;13,5,4

- EPS Division, Liquids Section, 15,7,16

- Graphite intercalation compounds, 16, 11/12,16

- Itinerant electron magnetism, 14,7,7

- Localization and interactions of electrons, 14, 7,6

- Materials science experiments under microgravity, 16, 7/8,14

- Molecular and atomic hydrogen under extreme conditions, 13,8/9,4 
(Condensed Matter Physics)

- Neutron spin echo spectroscopy, 16,4,1

- Nuclear methods in, 16,4,9

- in One dimension, 12,2,10

- Phase transitions, diffraction studies, marginal dimensionality, 16,9,1 - Nobel Prize in Physics 1982, 14,2,7

- Semiconductor -

- Deep levels, 14,7,9

- Films, MBE grown, surface studies of, 16,4,12

- Structures, localisation, interaction effects in, 16,7/8,1

- Structure of matter research in Italy, 13,4,8

Constitution and By-laws, EPS : see EPS ...

Cosmic Rays: What are they made of ? 15,2,12

Cosmology: see Astronomy ...

Council, EPS: see EPS ...

Crystals

- Incommensurate, 13,12,1

- Polymer, structure and morphology, 13,12,10

Data bases: searching for data, 12,4,6

Data communications in high energy physics, $14,2,4$

Data storage: optical, on discs, 12,7,11

Denmark : steady state of physics in, 16,5,15

Developing Countries

- of Europe, problems of physics in, 12, 10,11

- Physics teaching in, 14,8/9,9

- Third-World Academy of Sciences, 15,2,15

Diffusion, deterministic: a chaotic phenomenon, 15, 5,5

EGAS meeting, Liège 1982, 14,4,11

- Madrid 1983, 15,4,16

Electronic and Atomic Collisions

- Atomic and molecular collision physics, 12, 1,3

- Energetic ion-atom collisions, theory of, 16, 1,6

Electrons

- Localization and interactions, 14,7,6

- One-electron problem, new methods for, 12,5,4

- and Positrons, channelled, radiation of, 12, 12,1

- Relativistic, coherent radiation from, 16,7/8,10

- Spin-polarized, 14,2,9

EPS Associate Members

- BESSY, 15,6,1

- CERN

- $\quad$ - ISOLDE experiments, 12,4,1

- LEAR experiments, 15, 7,5

- LEP ground-breaking ceremony, 14, 10,12

- Proton-antiproton collider experiments, 14,10,1

- GEC, long-range research at, 14,5,5

- Geneva meeting 1982, 13,12.11

- JET

- $\quad$ - in August 1984, 15,8/9,7

- Opening ceremony, 15,5,16

- Scientific programme, $14,4,3$

- STL, Optical fibre technology at, 16, 11/12,4

- Winterthur meeting 1984, 15,5.14

EPS Constitution and By-laws, revision of, $12,12,12 ; 13,4,12 ; 13,12,12$;

EPS Council

$14,4,12 ; 15,4,2 ; 16,4,16$

- Berlin-West $1985,16,4,15 ; 16,5,5$

- Copenhagen 1983, 14,4,1

- Geneva 1982, 13,5,1

- Grenoble $1981,12,4,12 ; 12,5,1$

- Istanbul 1981, 12,10,3

- Prague 1984, 15,8/9,1

- Winterthur 1984, 15,4,1

EPS Executive Comm. statement on nuclear arms, 13, 7,12

EPS General Conference

- 5th (Istanbul 1981), 12,1,1; 12, 10,1

- 6th (Prague 1984), 14, 12,1; 14,6,1; 15,8/9,1; 16,5,16

EPS General Meeting of Members, $12,10,4 ; 15,8 / 9,2$

EPS Lecturer

- 1982 (I.F. Silvera), 13,4,12; 13,8/9,4

- 1984 (P.T. Matthews), 15,4,3

- 1985/6 (H. Haken), 16,9,16

EPS Letters Journal: see Europhysics Letters

EPS President

- J. Friedel, message, 13,4,1

- A.R. Mackintosh: rendez-vous Istanbul, 12,1,1

- $\quad$ - State of EPS, 12,8/9,1

- G.H. Stafford, message, $15,5,1 ; 15,8 / 9,2$

EPS Teaching abroad scheme, $13,2,7 ; 13,8 / 9,11 ; 15,6,12$

Euler, 1707-1783, 14, 10,6
European Communities, R,D and D support by, 14, 12,7

European Geophysical Society, 15,2,15

European Incoherent Scatter (EISCAT) Project, inauguration, 12, 10,5

European Physical Society: see EPS ...

Europhysics Letters, 16, 2,3; 16,6,1; 16,11/12,20

Fundamental constants and SI units, $15,7,8$

Galileo, Nobel and the $Z^{\circ}, 14,7,3$

Gamma-ray internal conversion, applications, 15,4,11

GEC, long-range research at, $14,5,5$

Heavy lons

- in Collisions, $12,1,6 ; 16,1,6$

- Reactions, 15, 1,10

Hewlett-Packard Europhysics Prize

- 1980: O.K. Andersen, 12,5,4

- 1982: K. von Klitzing, 13,4,2

- 1983: I. Silvera, 14,2,12

- 1984: G. Binnig, H. Rohrer, 15, 2,16; 15,5,16

- 1985: J. Als-Nielsen, M. Pepper, 16, 1,16; 16,4,11; 16, 7/8,1; 16,9

High Energy and Particle Physics

- Big detectors using BGO, 16,2,8

- Broken symmetries, 12,2,1

- Data communications in, 14,2,4

- Intermediate vector bosons, 14, 10,1

- ISOLDE experiments, 12,4,1

- LEAR experiments, 15,7,5

- LEP ground breaking ceremony, 14, 10,12

- Muons as probes in solids, 16,2,11

- Nobel Prize in Physics 1980, 12,2,1

$-1984,15,11 / 12,1$

- Parity non-conservation in heavy atoms, 16,2,1

- Proton-antiproton collider experiments, 14,101

- Three-nucleon force, 15,2,5

- Where do we stand?, 12,2,4

-Where is it going? $13,10,4$

Histories of science, $\mathbf{1 4 , 7 , 4}$

Hydrogen production by water-electrolysis, 12,8/9,9

Iceland, physics in, 16,6,15

ICFA (Int. Comm. for Future Accelerators), 16,6,13

International Facilities for Physics Research, 14,8/9,9

ISOLDE experiments at CERN, 12,4,1

Isotopes

- Anomalies in solar system, 14,2,1

- ISOLDE experiments, 12,4,1

- Separation by lasers, 12, 7,5

Italy, research on structure of matter in, 13,4,8

Japan Physical Society, collaboration with, 16,9,16 JET

- in August 1984, 15,8/9,7

- Official opening, 15,5,16

- Scientific programme, $14,4,3$

Lasers

- Biological and medical applications, 12,7,14

- Chemical vapour deposition induced by, 14, 10,9

- Dye, in quantum electronics, 13,10,10

- Free-electron, 13,4,5; 16,7/8,10

- Hollow-cathode, research on, 13,2,9

- Industrial applications, 12, 7,10; 14, 10,9

- Isotope separation by, 12,7,5

- Nobel Prize in Physics 1981, 13,2,6

- Optical storage on discs, 12,7,11

- Particle tracking by, 15,5,2

- Photochemistry applications, 12,7,4

- Physical principles, historical review, 12,7,1

- Plasma diagnostics, 12,7,12

- Special Issue, 12,7

LEAR experiments at CERN, 15, 7,5

Letters to the Editor

- Database Guides (N.B. Butterwick), 12,4,7

- Parity violation predictions (M.A., C. Bouchiat, D.N. Stacey), 16, 5,14

- Physics and culture (J.W.R. Fennema), 15,4,4

Low Temperature Physics

- Refrigeration to ultralow temperatures, 13,12,7

Macromolecular Physics

- Amphiphiles, micelles, microemulsions, 16,6,9

- Current problems, 14,8/9,1

- Polymer crystals (1982 Spain Conf.), 13, 12,10 


\section{Magnetism}

- Itinerant-electron type, 14,7,7

- Nijmegen high-field laboratory, 15, 11/12,13

- Permanent magnets, 13,7,5

- Spin-glasses, 14, 12,2

Metal Physics

- Cyclic plasticity in pure metals, 14, 1,2

- Heavy fermions, 16,9,14

- Intermetallic compounds, hydrogen in, 14,4,7

- Mechanical instabilities, 14, 12,10

- Metallic glasses, 14, 1,8

- Physical metallurgy, advances in, 14, 1,1

- Special Issue, 14,1

- Superconductivity and metallurgy, 14, 1,6

- Superplasticity of metals, 14,1,4

- Surfaces of metals, 14,1,10

Microscope, scanning-tunnelling (Winterthur meeting 1984), 15,5,14

Neutrinos in cosmology, 14,7,1

Neutron spin-echo spectroscopy, 16,4,1

Neutron stars, $12,1,5$

Nobel Prize in Physics

- 1980: J.W. Cronin, V.L. Fitch, 12,2,1

- 1981: N. Bloembergen, A. Schawlow, 13, 2,6; K. Siegbahn, 13,2,5

- 1982: K.G. Wilson, 14,2.7

- 1983: S. Chandrasekhar, W.A. Fowler, 14,12,5

- 1984: S. van der Meer, C. Rubbia, 15, 11/12,1

- 1985: K. von Klitzing, 16, 11/12,1

Nuclear magnetic resonance for tomography, 15,5,10

Nuclear Physics

- Atomic nucleus, short-range structure of nuclear matter, 14,5,1

- Gamma-ray internal conversion, applications, $15,4,11$

- Heavy-ion collisions, 12, 1,6: 16,1,6

- Heavy-ion reactions, 15, 1,10

- Methods for condensed matter studies, 16,4,9

- New concepts, challenges, 15,1,1

- Nuclear stability, 14,8/9,4

- Nuclei, rapidly rotating, $15,1,5$

spin vibrations in, 15, 1,2

- supersymmetry in, 15, 1,8

- synthesis of, at limits of nuclear stability, 14,8/9,4

- Pion as probe, $15,1,14$

- Radioactive ions, secondary beams of, 12,4,1

- Special Issue, 15,1

- Three-nucleon force, 15,2,5

- in early 1930s, $13,8 / 9,1$

\section{Obituaries}

- Sir John Adams, 15,4,15

- P.A.M. Dirac, 15, 11/12,16

- P.L. Kapitza, $15,5,8$

- A. Kastler, 15,4,14

- L.A.A. Thomas, 16, 2,16

Optical fibre technology at STL, 16,11/12,4

Optical storage on discs, $12,7,11$

Optics, inverse problems in, $13,5,6$

Particle Accelerators

- Accelerator mass spectrometry of radioisotopes, 15,2,1

- ICFA (Int. Comm. for Future Accelerators), 16,6,13

- Memories from childhood of, 15,2,9

- for Single-atom counting, 15,2,1

Physics Education

- Helping developing countries, 12, 10,11; 14,8/9,9

- at Secondary school level, 12,2,8

- Teaching abroad scheme, $13,2,7 ; 13,8 / 9,11 ; 15,6,12$

- in Turkey, 13,8/9,11

Physics of the Ocean, 16,11/12,13

Physics Research (see also Research in Europe)

- in Denmark, 16,5,15

- in Iceland, 16, 6,15

- International facilities for, 14,8/9,9

- in Italy, on structure of matter, 13,4,8

- Problems in Europe's developing countries, 12, 10,11

- in Turkey, 12,8/9,2

Physics and Society

- Career outlook for physicists in Europe, 12,12,8

- Helping developing countries, 14,8/9,9

- Interaction, 12,2,7

- Problems in Europe's developing countries, 12, 10,11

- Radioactive waste disposal, 12,5,9

- Training and jobs for Ph.D.s, 13, 10,1
Plasma Physics (see also Thermonuclear Fusion)

- Atoms in highly ionized states, 12,1,1

- Computations, 12,12,4

- Diagnostics using lasers, 12, 7,12

- EPS Division meeting, Moscow 1981, 12,12,11

- Magnetic confinement, 15,4,5

- Particle tracking by lasers, 15,5,2

- Stellarators, present and future, 13,8/9,7

- Turbulence, self-organization of, 13,2,1

Publications

- Annual Reviews Inc., reduced subscriptions, 13,8/9,12

- Europhysics Letters, 16, 2,3; 16,6,1; 16,11/12,20

- Institute of Physics, reduced subscriptions, 15, 11/12,16

- Physics periodicals in Europe, 13,2,11

- Reduced subscriptions for IOMs, 13, 10,12

- Swiss Physical Society, Helv. Phys. Acta changes, 13,5,3

Quantum Electronics (see also Lasers)

- Changes in emphasis, 12,2,5

- Excited states, multiresonant optical nonlinearities, 12,5,12

- Munich Conference 1982, 13,10,10

- Nobel Prize in Physics 1981, 13,2,6

Quantum Hall effect, 13,4,2

- Nobel Prize in Physics 1985, 16, 11/12,

Quantum Optics, 9th Summer School, Poland 1981, 13,2,8

Radioactive waste disposal, 12,5,9

Radioisotopes, Long-lived, accelerator mass spectrometry for, 15,2,1

- Secondary beams of, 12,4,1

Rare earths, mixed-valence compounds, 13,7,9

Research in Europe (see also Physics Research)

- Priorities of funding agencies, 14, 10,8

- R,D and D support criteria of European Communities, 14,12,7

Semiconductor -

- Deep levels, 14, 7,9

- Films, MBE grown, surface studies of, 16,4,12

- Structures, localisation, interaction effects in, 16,7/8,1

SI Units and fundamental constants, 15,7,8

Smail Particles

- in Gas suspension, 15, 11/12,6

- Laser tracking of, 15, 5,4

Solar Physics

- EPS Section, 13,1,1; 12,8/9,12

- European developments in, 13,1,1

- Magnetic face of the Sun, 13,1,10

- Solar corona, physics of, 13,1,8

- Solar cycle, origin of, 13,1,5

- Solar interior, diagnostics of, 13,1,3

- Solar and stellar magnetic fields, 13,12,6

- Solar system, isotopic anomalies in, 14,2,1

- Special Issue, 13,

Solid State Physics (see also Condensed Matter Physics)

- Crystals, Incommensurate, 13, 12,1

- Polymer, structure and morphology, 13, 12,10

- Disordered systems, macroscopic quantum effects in, 15, 11/12,3

- Ferroelectrics, dielectric spectroscopy of soft modes, 15,7,1

- Intercalation compounds, 13,2,8

- Metal physics (Special Issue), 14, 1

- Muons as probes ( $\mu$ SR), 16,2,11

- One-electron problem, new methods for, 12,5,4

- Phase transition theory, 14,2,7

- Rare earths, mixed-valence components of, 13,7,9

- Recent developments, 12,1,7

- Superconductivity in organic solids, 14,5,7

Spacelab 1 : materials experiments under microgravity, 16,7/8,14

Spectroscopy

- Dielectric, of soft modes in ferroelectrics, 15,7,1

- of Dimers, and vibrational predissociation, 16,1,9

- Electron, Nobel Prize in Physics 1981, 13,2,5

- Mōssbauer, gamma-ray internal conversion for, 15,4,11

- Neutron spin echo, 16,4,1

- Novel types of, 15, 5,14

- Uitrasonic, of superfluid ${ }^{3} \mathrm{He}, 16,9,9$

Spin-glasses, 14,12,2

STL, Optical fibre technology at, 16, 11/12,4

Superconductivity

- and Metallurgy, 14, 1,6

- in Organic solids, 14,5

Superfluid ${ }^{3} \mathrm{He}$

- Rotating, vortices in, $\mathbf{1 5 , 6 , 1 3}$

- Ultrasonic spectroscopy of, 16,9,9 
Surfaces and Interfaces

- Structure investigation by He diffraction, 12,10,6

- Studies of MBE grown semiconductor films, 16,4,12

- Surface physics at BESSY, 15,6,1

- Surface reaction dynamics, 13,5.9

- Surface science, 13,12,5

Swiss Physical Society, changes in Helv. Phys. Acta, 13,5,3 Synchrotron Radiation

- at BESSY, for surface physics, 15,6,

- Relativistic electrons and coherent radiation, 16,7/8,10

Thermonuclear Fusion

- JET, Official opening, 15,5,16

- - Scientific programme, 14,4,3

- Neutral injection heating in devices for, 16,4,5

- Reactor engineering problems, 16,5,11

Reversed field pinch in magnetic fusion, 15,4,5
- RF driven currents in devices for, 15,8/9,6

- Stellarators, 13,8/9,7

- Tandem mirror approach to magnetic fusion, 12,8/9,4

Third-World Academy of Sciences, 15,2,15

Training and jobs for physics and engineering Ph.D.s, 13, 10,1

Trends in Physics (EPS General Conf.)

- Istanbul 1981, 12, 1,1:12,10,1

- Prague $1984,14,6,1 ; 14,12,1 ; 15,8 / 9,3 ; 16,5,16$

Turbulence, self-organization of, 13,2,1

Turkey, Physics in, 12,8/9,2

- Teaching in, 13,8/9,11

Ultrasound

- for Biological tissue characterisation, 15,8/9,12

- for Spectroscopy of superfluid ${ }^{3} \mathrm{He}, 16,9,9$

UNESCO coupons, $14,10,12$
W. Allison, 13, 12,5

J. Als-Nielsen, 16,9,1

I. Ambar, 16, 11/12,13

P. Ambruster, 14,8/9,4

O.K. Andersen, 12,5,4

A. Andreoni, 12,7,14

H. Andrillat, 12,8/9,7

J. Audouze, 14, 12,5

J. Auth, 12, 12,10

M.Ya. Azbel, 15, 11/12,3

P. Bak, 14,2.7

D.E. Baldwin, 12,8/9,4

F.J. Baltá-Calleja, 13, 12,10

H.P. Baltes, 13,5,6

D. Bäuerle, 14, 10,9

K. Bechgaard, 14,5,7

F.S. Becker, 12,7,5

R. Behn, 12,7,12

K. Bergmann, 12, 1,3

E. Bernabeu, 14,4,11

K. Bethge, 16,4,9

A. Bewersdorff, 16, 7/8,14

G.F. Bignami, 13,7,1

M. Billardon, 16,7/8,10

R. Billinge, 15, 11/12,1

D. Biskamp, 12,12,4

P. Bochsier, 14,2,

G. Born, 12,2,9

E. Borsella, 16,1,2

F. Bosch, 12,1,3

C.E. Bottani, 14, 12,10

L. Bottinelli, 15, 11/12,10

C. Bouchiat, 16,5,14

M.A. Bouchiat, 16,5,14

G. Bouwhuis, 12,7,11

J.J.M. Braat, 12,7,11

A.M. Bradshaw, 15,6,1

S. Bratos, 15, 7,16

W. Braun, 15,6,

G. Brianti, 14, 10,1

J.S. Briggs, 16,1,6

J. Brossel, 15,4,14

D.V. Bugg, 15, 7,5

H. Burtscher, 15, 11/12,6

N.B. Butterwick, 12,47

G. Caglioti, 14, 12,10

R. Cahn, 14, 1,4

C.J. Cesarsky, 12, 1,5; 15,2,12

M. Chabre, 16,5,1

D.S. Chemla, $12,2,5 ; 12,5,12$

C. Chiuderi, 13, 1,8

M. Chown, 16,11/12,4

F. Ciatti, 12,4,8

R.M.J. Cotterill, $14,1,1 ; 14,1,8$

L. Csillag, 13,2,9

R. Cubeddu, 12,7,14

W. Czaja, 14, 7,6

P.J. Dean, 15, 7,9

V. Degiorgio, 16,6,9

J. Désesquelles, $15,4,16$

J.T. Devreese, 12, 1,7; 13,2,12;

$13,5,5 ; 16,1,6 ; 16,11 / 12,3$

D.P. Di Vincenzo, $15,11 / 12,3$

\section{CONTRIBUTORS}

E.R. Dobbs, $16,9,9$

H. Doubre, $15,1,10$

O. Dragoun, $15,4,11$

H.W. Drawin, 12, 1,

P. Elleaume, $13,4,5$

J. Ellis, 16, 9,6

F. Engelmann, 12, 12,11

D.J. Fabian, 14, 1,10

R. Fantoni, 16, 1,2

Y. Farge, 13,4,5

E.A. Fellmann, 14, 10,6

J.W.R. Fennema, 15,4,4

B. Feuerbacher, 16,7/8,14

M. Fink, 15,8/9,12

P.E. Fricker, 14, 10,8

J. Friedel, 13,4, $;$ 13, 10 ,

P. Fulde, 16,9,14

C. Gaarde, 15, 1,2

E. Gabathuler, 14, 10,

J.D. Garrett, $15,1,5$

F.D. Gault, 12,4,6

T. Geisel, 15,5,5

F.A. Gianturco, 12,1,3; 16, 1,

A. Giardini-Guidoni, 16, 1,2

A. Gibson, 14,4,3

W. Glöckle, 15,2,5

Ch. Gnehm, 13, 7,5

C. Gormezano, 15,8/9,6

D. Gough, 13,1,3

L. Gouguenheim, 15, 11/12,10

M. Grätzel, 12,7,4

G. Grieger, 16,5,11

A. Guinier, $14,7,4$

G.B. Hagemann, 15, 1,5

P.G. Hansen, 12,4,1

A. Hartstein, 15, 11/12,3

A. Hasegawa, 13,21

V. Heine, 13,5.4

B. Herskind, 15, 1,5

M.D. Hill, 12,5,9

M.G.N. Hine, 14,2,4

F. Hottier, 15,8/9,12

F. lachello, 15, 18

Y. Imry, 16, 11/12,

E. Inönü, 12,8/9,2

ISOLDE Collaboration, 12,4,

G. Israel, 12, 10,9

F. James, 12, 1,11

A. Janner, $12,2,9 ; 13,12$,

M. Jánossy, 13,2,9

T. Janssen, 13,12,

C. Jarlskog, 12,2,1

D. Jérome, 14,5,7

B. Jonson, $12,4,1$

J.L. Jorda, 14, 1,6

C. Jordan, 12,8/9,12

B.A. Joyce, $16,4,12$

J. Kaczer, 14, 12,1

F. Kaczmarek, 15,6,12

K. Kalyanasundaram, 12,7,4

H. Kamimura, 13,2,8
B. Kasemo, 13,5,9

T. Katila, 15, 7,11

H.H. Kausch, $14,8 / 9,1$

A. Keller, 14,8/9,1

K. von Klitzing, 13,4,2

K.L. Kompa, 12,7,5

V. Kose, 15, 7,8

G.V. Kozlov, 15,7,

J.A. Krumhansl, 12, 2,10

W. Kündig, 16,2,11

J. Laberrigue, 14,7,4

J.M. Laget, 14, 5,

P.K. Larsen, 16,4,12

P. Lecoq, 16,2,8

R. Lefebvre, 16,2,4

P.R. Locher, 15, 5,10

W.O. Lock, 16,6,13

P. Lomas, 16,4,5

B.I. Lundqvist, $13,5,9$

A.R. Mackintosh, 12, 1,1; 12,8/9,1; 16, 5,15

A. Magerl, 16,11/12,16

P. Maltby, 13,1,

J.P. Martin, 16,2,8

Ph.A. Martin, 13,5,3

T. Mayer-Kuckuk, 15, 1,1

W. Mehlhorn, 12, 7,15

P.F. Meier, 16,2,11

J.-P. Meyer, 15,2,12

F. Mezei, 16,4,1

J.J.H. Miller, 13, 12,6

P.D. Morgan, 12,7,12

F.M. Mueller, 13,5,5

J. Muller, 14,1,6

E. Müller-Hartmann, 13, 7,9

J.A. Mydosh, 14, 12,2

H.W. Myron, 15,11/12,13

W.S. Newman, $14,7,3$

S. Ortolani, 15,4,5

M. Paillon, 14, 12,7

D.H. Parkinson, 12,2,7

M. Pepper, 16, 7/8 1

Y. Petroff, 16,7/8,10

J. Petzelt, 15, 7,1

G.R. Plattner, 13,5,3

F. Pobell, 13, 12,7

G. Preparata, 12,2,4

J. Reuss, 16,1,9

T.M. Rice, 14, 7,6

K.R. Richmond, 16, 11/12,4

K.H. Rieder, $12,10,6$
C. Rizzuto, $13,4,8$

I.W. Roxburgh, $12,8 / 9,12$

K. Rózsa, 13,2,9

C.A. Sacchi, 12,7,14

B. Sandell, 13,8/9,1

P.U. Sauer, 15, 2,5

L. Schlapbach, 14,4,7

A. Schmidt-Ott, 15, 11/12,6

M. Schneegans, 16,2,8

H. Schopper, 13, 10,4

M. Schüssler, 13, 1,5; 13, 12,6

D.G. Scotter, 14,5,5

M.J. Seaton, 16, 1,12

E.N. Shaw, 15,4,15

H.C. Siegmann, 14,2,

M.R. Siegrist, 12, 7,12

T.I. Sigfusson, 16,6,15

I.F. Silvera, 13,8/9,4

H. Smith, 16,5,15

S.D. Smith, 13,2,

I.J. Spalding, 12,7,10

D.N. Stacey, 16,2, ?; $16,5,14$

G.H. Stafford, 15, 5, $1 ; 15,8 / 9,2$

R.P. van Stapele, 15, 5,10

R.A. Stern, 15,5,2

S. Stolte, 16, 1,9

S. Stucki, 12,8/9,9

O. Svelto, 12,7,1

J.-P. Swings, 15,4,8

K. Taulbjerg, 16,1,6

R.J. Tayler, 14,7.1

F.K. Thielemann, 16,7/8,5

J.M. Thomas, 13,2,5

N. Vinh Mau, 12, 1,6

M. Vivargent, 16,2,8

A.A. Volkov, 15, 7,1

G.E. Volovik, 15,6,13

E.T.S. Walton, $13,8 / 9$

R. Wedell, 12,12,1

G. Werth, 13,2,8

C. White, 14, 12,7

R. Wideröe, 15,29

C. Wilkin, 15, 1,14

B.L.H. Wilson, 16,5,9

A.T. Winter, $14,1,2$

H. Wobig, 13,8/9,7

H. Wöhl, 13, 12,6

E.P. Wohlfarth, 14,7,7

P.M. de Wolff, 13,12,

W. Wölfli, 15,2,1

J.J. Wynne, 13, 10,10

R. Zelazny, 12,12,11

C. Zwaan, 13, 1,10
A cumulative index of Europhysics News covering the years 1976-1980 is in preparation and will be available early in 1986 from the

EPS Secretariat, POB 69

CH - 1213 Petit-Lancy 2

Switzerland 